\title{
A Statistical Investigation to Monitor and Understand Atmospheric CFC Decline with the Spatial-longitudinal Bent-cable Model
}

\author{
Shahedul A. Khan ${ }^{1}$, Masud Rana ${ }^{1}$, Longhai Li $^{1} \&$ Joel A. Dubin ${ }^{2}$ \\ ${ }^{1}$ Department of Mathematics and Statistics, University of Saskatchewan, Saskatoon SK S7N 5E6, Canada \\ 2 Department of Statistics and Actuarial Science \& School of Public Health and Health Systems, University of \\ Waterloo, Waterloo, Ontario N2L 3G1, Canada \\ Correspondence: Masud Rana, Department of Mathematics and Statistics, University of Saskatchewan, Saskatoon \\ SK S7N 5E6, Canada. Tel: 1-306-966-2297. E-mail: mdr091 @ mail.usask.ca
}

Received: May 20, 2012 Accepted: June 7, 2012 Online Published: July 30, 2012

doi:10.5539/ijsp.v1n2p56 URL: http://dx.doi.org/10.5539/ijsp.v1n2p56

The research is partially supported by the University of Saskatchewan through President's NSERC grant to S. A. Khan, and by NSERC through discovery grants each to L. Li and J. A. Dubin

\begin{abstract}
Concerns about the concentrations of chlorofluorocarbons (CFCs) in the atmosphere are based on their effects on the ozone layer by catalytically destroying ozone. The recent steady decline in atmospheric concentration of CFC could be a direct result of the Montréal Protocol's ban on CFC products, in effect since 1989. However, CFCs have long atmospheric residence times because of their low chemical reactivity, and as a consequence have already been distributed globally. To study the spatial effects and extent of the decline, we apply the proposed spatial-longitudinal bent-cable model to CFC data observed over a global detection network. The bent cable is a parametric regression model to study data that exhibits a trend change. It comprises two linear segments to describe the incoming and the outgoing phases, joined by a quadratic bend to model the transition period. For spatial longitudinal data, measurements taken over time are nested within spatially dependent locations. Here, it is useful to extend the existing longitudinal bent-cable regression to handle spatial effects. We do so in a hierarchical Bayesian framework by allowing the error terms to be correlated across space. The methodology is illustrated with applications to $\mathrm{CFC}-11$ and 12 data. Our analysis reveals that: (a) there is a strong spatial relationship among all the monitoring locations across the globe; (b) both CFC-11 and CFC-12 increased significantly before entering into a transition zone; (c) after completing the transition, CFC-11 has been decreasing significantly from the atmosphere, but a slow (insignificant) decrease for CFC-12 is observed; and (d) it may take almost 5 times longer to diminish CFC-12 from the atmosphere compared to CFC-11.
\end{abstract}

Keywords: atmospheric ozone depletion, Bayesian inference, bent cable regression, chlorofluorocarbon, longitudinal data, spatial effects

\section{Introduction}

Atmospheric ozone layer protects all living things from the detrimental effects of ultraviolet (UV) radiation from the sun. In particular, about $90 \%$ of the total atmospheric ozone lies in the stratosphere, which is the major source of blocking the harmful UV radiation to reach in the earth's surface (Struijs et al., 2010). The impact of thinning ozone layer caused by chlorouorocarbons (CFCs) on human health and environment is now globally recognized (Han, Kennedy, Mackie, \& Dlugogorski, 2010; Struijs et al., 2010); negative effects of increased UV exposure include skin cancer and cataracts to humans, and irreversible damages to plants and drifting organisms (e.g. animals, plants, archaea, bacteria) in the ocean's photic-zone (Khan, Chiu, \& Dubin, 2009).

CFCs comprise about $90 \%$ of the Ozone Depleting Substances (ODSs) in the atmosphere, and more than $80 \%$ of these are accounted by CFC-11 and CFC-12, the most abundant CFCs in the atmosphere (Moulijn, Makkee, Wiersma, \& van de Sandt, 2000). These CFCs are sources of chlorine to the atmosphere, which catalytically destroy stratospheric ozone (Molina \& Rowland, 1974). In particular, each chlorine atom can break down an average 
of 100, 000 ozone molecules during it's 1-2 years atmospheric lifetime. Furthermore, they are strong infrared absorbers in the atmospheric window region and are very potent greenhouse gases (Zhang et al., 2010). The 1987 Montréal Protocol on Substances That Deplete Ozone Layer (World Meteorological Organisation, 2011) formally recognized the significant threats of the ODSs, and provided a mechanism to phase-out global production and consumption of CFCs along with other ODSs.

Since the Montréal protocol came into effect, emissions of CFCs have changed dramatically, and their atmospheric concentrations have either leveled off or decreased. Relative to the corresponding peak values in the late 1980s, the annual aggregated production and consumption of ODSs have declined by $95 \%$, and annual emissions by $82 \%$ of which around $80 \%$ are accounted by CFCs (Ravishankara et al., 2008). Although the production of CFCs has decreased substantially and is expected to continue to decrease in the future, the amount of CFCs in existing products and equipments has the potential to make an important contribution to future CFC emissions (Zhang et al., 2010). In addition, due to their extended lifetimes (45 years for CFC-11 and 100 years for CFC-12 (World Meteorological Organisation, 2011)), CFCs can stay in the upper atmosphere for a substantially long period of time. During that time, winds spread them across the world. Consequently, the atmospheric concentration of CFCs is now a global concern, and it is hypothesized that they have already been distributed globally (i.e., all areas around the globe are neighbours with respect to the CFC concentration in the atmosphere). So, it is important to understand the spatial distribution of the CFC concentration around the globe by fitting an appropriate statistical model.

CFCs are monitored from different stations across the globe. Each station constitutes an individual curve for CFC trend, which is different from the others due to the differences in instruments, sampling techniques, exposure to wind, actual levels of the CFC during measurement, and so on. Therefore, the appropriate statistical method to analyze these data should involve a longitudinal model which can characterize a trend change over time. Chiu, Lockhart and Routledge (2006) and Chiu and Lockhart (2010) developed a special changepoint model to analyze a single profile that exhibits a trend change. The model is parsimonious with respect to the number of parameters, and appealing due to its simple structure, flexibility and interpretability. It comprises two linear segments (incoming and outgoing) joined by a quadratic bend. There are five parameters: an intercept and a slope for the incoming phase, two parameters to characterize the center and the half-width of the bend, and a slope for the outgoing phase. Khan et al. (2009) proposed an extension of the bent-cable model for longitudinal data, but with virtually no methodological details. They addressed the global concern of CFC-11 by fitting their longitudinal bent-cable regression model. However, they did not take into account spatial effects, an important aspect to understand the atmospheric distribution of $\mathrm{CFCs}$ around the globe, and did not investigate CFC-12, not to mention had considered a shorter period of follow-up for CFC-11.

Accounting for the spatial effects, we develop a statistical framework for the longitudinal bent-cable model to analyze both the CFC-11 and 12 data. We will address the following questions statistically by fitting our proposed spatial-longitudinal bent-cable model: (1) What is the spatial distribution of the CFC concentration around the globe? (2) What was the time point at which the CFC concentration took a downturn from an increasing trend? (3) Has the concentration of CFC been decreasing significantly after the transition? (4) Are there any differences and/or similarities in the atmospheric concentration of CFCs in different parts of the world? Addressing these questions could be useful not only to policy makers, but also for human awareness. For example, (a) through the estimation of the strength and extent of the distribution of CFCs around the globe, our analysis will reveal whether the concern about CFCs is global or regional, and (b) through the estimation of the transition and the rate by which CFCs have been declining from the atmosphere, our analysis will provide insight about the extent of the ozone destruction process, which, in turn, will convey information regarding precautions against the UV radiation. In a broader sense, we will also comment on the effectiveness of the Montreal Protocol in reducing the use of CFCs.

In Section 2, we describe the CFC data. In Section 3, we present our modeling framework to account for spatial effects in the longitudinal bent-cable model. This is all constructed under a Bayesian framework (Section 4). We then apply our method to the CFC-11 and CFC-12 data to address the aforementioned questions (Sections 5). In Section 6, we present a simulation study to demonstrate the performance of our proposed methodology. We summarize our findings in Section 7.

\section{CFC Data}

CFCs are monitored from different stations across the globe by the Global Monitoring Division of the National Oceanic and Atmospheric Administration (NOAA/ESRL halocarbons group, http://www.esrl.noaa.gov/gmd/), and Atmospheric Lifetime Experiment/Global Atmospheric Gases Experiment/Advanced Global Atmospheric Gases 
Experiment (ALE/GAGE/AGAGE, http://agage.eas.gatech.edu/data_archive/) program sponsored by NASA. Both these programs currently use gas chromatographic (GC) technique (known as in situ program) to measure CFCs. Below we summarize the process (also see Figure 1) as described in the NOAA/ESRL website http://www.esrl.noaa.gov/gmd/hats/insitu/insitu.html.

The process of measuring CFCs begins by drawing outside air through a sampling line using a pump. The air then enters the gas sampling valve, where it flushes the valve for about five minutes. This process leads the sample to bleed down to ambient pressure. The GC analysis begins by injecting the sample into a separation column. Carrier gas (usually nitrogen or a mixture of methane and argon) is then used to sweep the sample through the separation process. Each type of molecule has a different rate of progression. Therefore, by examining the time to reach the end of the column, different components of the sample can be separated. An electron capture detector is used to produce chromatogram, the dip of which is considered as the measure of the amount of chemical present.

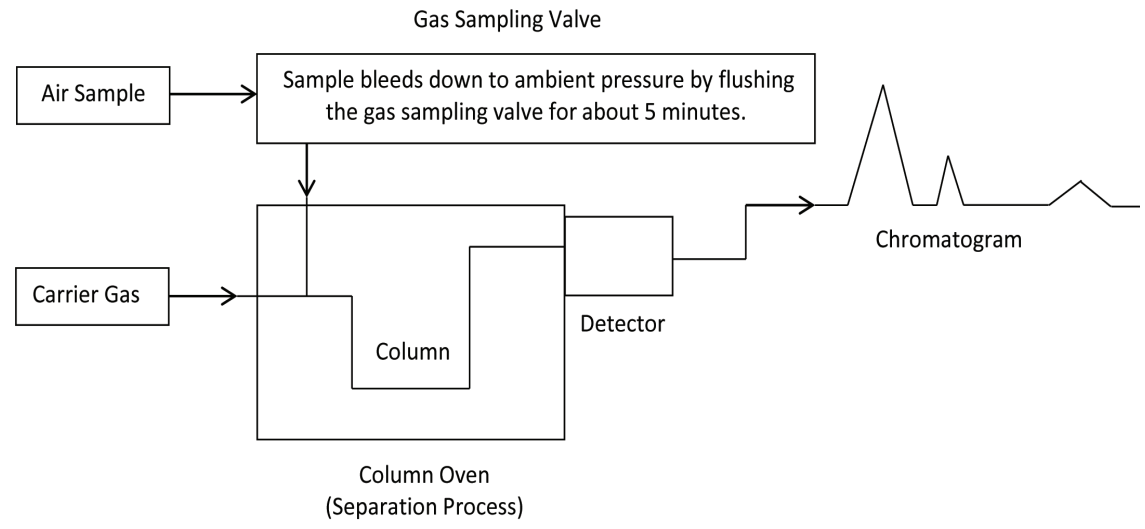

Figure 1. The analytic technique of separation and detection of CFCs using gas chromatograph

The in situ program of NOAA/ESRL started in 1987. The original program is called Radiatively Important Trace Species (RITS) program. In its complete form, RITS system consisted of two GCs and three columns, though only one GC (Hewlett-Packard 5890) and two columns (A and B) were used to measure CFCs. Each of the columns was loaded with a unique packing material: Porasil B for Channel A and OV-101 for Channel B. A 5\% mixture of Methane $\left(\mathrm{CH}_{4}\right)$ in Argon (called P5) and Nitrogen $\left(\mathrm{N}_{2}\right)$ were used as carrier gases in Channels A and B, respectively. The RITS GCs were deployed at five NOAA observatories: (1) Barrow, Alaska, (2) Cape Matatula, American Samoa, (3) Mauna Loa, Hawaii, (4) South Pole, Antarctica, and (5) Niwot Ridge, Colorado. However, widespread use of the replacement compounds to the CFCs prompted improvement of the RITS program. A new generation of GC called Chromatograph for Atmospheric Trace Species (CATS) was developed in 1998. The CATS GC consists of four channels and are capable of measuring nine other compounds in addition to the gases measured by RITS. This system replaced the RITS in situ instrumentation in 1998, and has been used to measure CFCs ever since.

Table 1. Geographical locations (latitude, longitude, and elevation in meter above sea level (masl)) of the stations and the instrumentations used to record data

\begin{tabular}{lcccc}
\hline & Latitude & Longitude & Elevation (in masl) & Instrumentation \\
\hline Barrow, Alaska & 71.32 & -156.60 & 11 & RITS/CATS \\
Cape Matatula, American Samoa & -14.24 & -170.57 & 42 & RITS/CATS \\
Mauna Loa, Hawaii & 19.54 & -155.58 & 3397 & RITS/CATS \\
South Pole, Antarctica & -89.98 & -24.80 & 2,810 & RITS/CATS \\
Niwot Ridge, Colorado & 40.04 & -105.54 & 3,018 & RITS/CATS \\
Mace Head, Ireland & 53.33 & -9.90 & 5 & GAGE/AGAGE \\
Cape Grim, Tasmania & -40.68 & 144.68 & 94 & GAGE/AGAGE \\
Ragged Point, Barbados & 13.17 & -59.43 & 45 & GAGE/AGAGE \\
\hline
\end{tabular}

The ALE/GAGE/AGAGE program (Prinn et al., 2000) consists of three phases corresponding to advances and 
upgrades in GC techniques: ALE (began in 1978), GAGE (began between 1981 and 1985), and AGAGE (began between 1993 and 1996). Under this program, CFCs are measured from stations located in (6) Mace Head, Ireland, (7) Cape Grim, Tasmania, (8) Ragged Point, Barbados, (9) Cape Matatula, American Samoa, and (10) Trinidad Head, California.

We take into account monthly mean CFC data based upon two considerations: (a) the availability of complete data, and (b) the necessity of a sufficiently long study period to capture the trend change over time. For (a), we choose eight monitoring stations (stations 1-8) for which data are available until September of 2010. Although the CFC monitoring process began in the late 1970's for some stations, complete data for all stations are not available until January of 1988. Therefore, we choose a study period beginning from January of 1988 to September of 2010 (273 months), which is also long enough to observe the trend change over time. Table 2 summarizes the geographical locations of the stations and the instrumentations used to record data.

Table 2. Model comparison for the CFC data using DICs

\begin{tabular}{lcc}
\hline Spatial & \multicolumn{2}{c}{ DIC } \\
Configuration & CFC-12 & CFC-11 \\
\hline Model 1 & 10,681 & 7,899 \\
Model 2 & 9,428 & 7,586 \\
Model 3 & 8,935 & 7,546 \\
Model 4 & 8,291 & 7,379 \\
\hline
\end{tabular}

\section{Spatial-longitudinal Bent-cable Model}

We index the stations by $i=1,2, \ldots, m$ and the months by $j=1,2, \ldots, n$. We model the CFC measurement for the $i^{\text {th }}$ station at time $t_{i j}$, denoted by $y_{i j}$, by the bent-cable regression model

$$
y_{i j}=f\left(t_{i j}, \boldsymbol{\theta}_{i}\right)+\epsilon_{i j},
$$

where the bent-cable function $f\left(t_{i j}, \boldsymbol{\theta}_{i}\right)$ is defined by

$$
f\left(t_{i j}, \boldsymbol{\theta}_{i}\right)=\beta_{0 i}+\beta_{1 i} t_{i j}+\beta_{2 i}\left[\frac{\left(t_{i j}-\tau_{i}+\gamma_{i}\right)^{2}}{4 \gamma_{i}} \mathbf{1}\left\{\left|t_{i j}-\tau_{i}\right| \leq \gamma_{i}\right\}+\left(t_{i j}-\tau_{i}\right) \mathbf{1}\left\{t_{i j}-\tau_{i}>\gamma_{i}\right\}\right]
$$

with $\boldsymbol{\theta}_{i}=\left(\boldsymbol{\beta}_{i}^{\prime}, \boldsymbol{\alpha}_{i}^{\prime}\right)^{\prime}, \boldsymbol{\beta}_{i}=\left(\beta_{0 i}, \beta_{1 i}, \beta_{2 i}\right)^{\prime}$ and $\boldsymbol{\alpha}_{i}=\left(\gamma_{i}, \tau_{i}\right)^{\prime}$. As illustrated in Figure $2, \beta_{0 i}$ and $\beta_{1 i}$ are, respectively, the intercept and slope of the incoming phase; $\beta_{1 i}+\beta_{2 i}$ is the slope of the outgoing phase; and $\gamma_{i}$ and $\tau_{i}$, the halfwidth and center of the bend, respectively, characterize the transition period. Note that the beginning and end of a transition can be represented by $\tau_{i}-\gamma_{i}$ and $\tau_{i}+\gamma_{i}$, respectively. Chiu \& Lockhart (2010) also defined the critical time point (CTP) at which the bent cable changes direction (i.e., takes a downturn from an increasing trend, and vice versa) by $\tau_{i}-\gamma_{i}-2 \beta_{1 i} \gamma_{i} / \beta_{2 i}$.

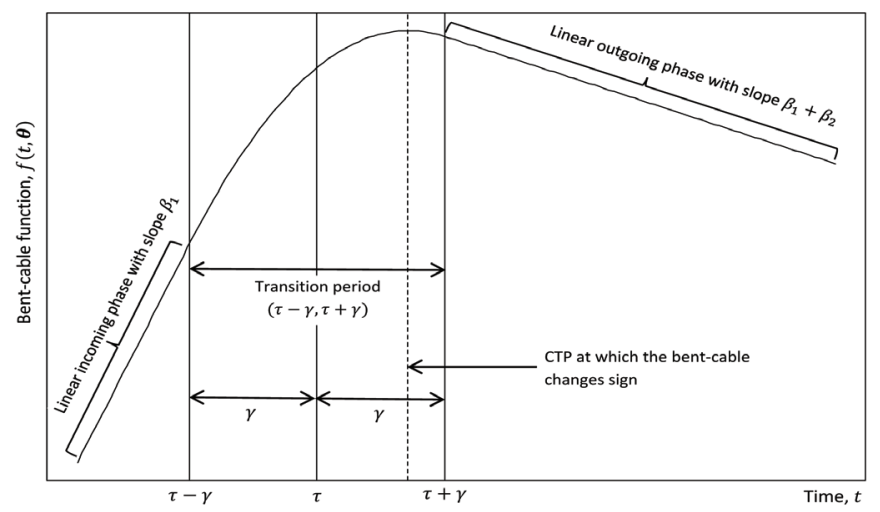

Figure 2. A graphical representation of the bent-cable function. The trend change is modeled by a quadratic bend characterizing the transition period $(\tau-\gamma, \tau+\gamma)$. Two linear models with slopes $\beta_{1}$ and $\beta_{1}+\beta_{2}$ characterize the incoming and outgoing phases. The time point at which the cable takes a downturn from the increasing trend is defined by CTP $=\tau-\gamma-2 \beta \gamma / \beta$, and is marked by the dotted vertical line 
To take into account the spatial effects, we model the error component as follows:

$$
\epsilon_{i j}=\delta \sum_{\substack{k=1 \\ k \neq i}}^{m} w_{i k} \epsilon_{k j}+v_{i j},
$$

where $\delta$ is the spatial autocorrelation coefficient that measures the strength of spatial association, $\mathbb{W}=\left(w_{i k}\right)_{m \times m}$ is the spatial weight matrix that describes the spatial arrangement of the locations (stations), and $v_{i j} \sim N\left(0, \sigma_{i}^{2}\right)$ is the random error component. Here, $w_{i k}$ is assumed known representing the a priori assumption about the spatial relationship between stations $i$ and $k$, with all the diagonal elements $w_{i i}$ set to zeros. The matrix $\mathbb{W}$ is typically normalized in an attempt to constrain $\delta$ in the interval $(-1,+1)$ (Elhorst, 2010). One such normalization technique is to divide the elements of $\mathbb{W}$ by its largest characteristic root. Note that $\mathbb{W}=\mathbb{I}$, where $\mathbb{I}$ is the identity matrix, implies no spatial effects, and constitutes the longitudinal bent-cable model of Khan et al. (2009).

Equations (1)-(3) constitute our spatial-longitudinal bent-cable model. The Bayesian hierarchical formulation of the model involves three levels. The first level (Level 1 or within-individual level) characterizes an individual trajectory by taking into account correlation and variation among the repeated measurements over time. The second level (Level 2 or between-individual level) models the station-specific regression coefficients $\boldsymbol{\theta}_{i}$ 's to take into account different patterns manifested by different trajectories. The third level (Level 3) quantifies prior information for the random quantities in Levels 1 and 2. Let $\mathbf{y}_{i}=\left(y_{i 1}, y_{i 2}, \ldots, y_{i n}\right), \mathbf{y}=\left(\mathbf{y}_{1}^{\prime}, \mathbf{y}_{2}^{\prime}, \ldots, \mathbf{y}_{m}^{\prime}\right)^{\prime}$, and $f_{i j} \equiv f\left(t_{i j}, \boldsymbol{\theta}_{i}\right)$. Also, let $\Theta$ be the vector of all the model parameters collectively. Then, based on our choices of the distributions for the relevant quantities, the hierarchical formulation of the model can be expressed as

$$
\left.\begin{array}{c}
g(\mathbf{y} \mid \Theta)=(2 \pi)^{-\frac{m n}{2}}\left|\mathbb{I}_{m}-\delta \mathbb{W}\right|^{n}\left\{\prod_{i=1}^{m}\left(\sigma_{i}^{-2}\right)^{\frac{n}{2}}\right\} \exp \left\{-\frac{1}{2} \sum_{i=1}^{m} \sum_{j=1}^{n}\left[\left(y_{i j}-\delta \sum_{k \neq i}^{m} w_{i k} y_{k j}\right)-\left(f_{i j}-\delta \sum_{k \neq i}^{m} w_{i k} f_{k j}\right)\right]^{2}\right\}, \\
{\left[\boldsymbol{\beta}_{i} \mid \boldsymbol{\mu}_{\beta}, \Sigma_{\beta}\right] \sim N_{3}\left(\boldsymbol{\mu}_{\beta}, \Sigma_{\beta}\right),\left[\boldsymbol{\alpha}_{i} \mid \boldsymbol{\mu}_{\alpha}, \Sigma_{\alpha}\right] \sim L N_{2}\left(\boldsymbol{\mu}_{\alpha}, \Sigma_{\alpha}\right),} \\
{\left[\boldsymbol{\mu}_{\beta} \mid \mathbf{h}_{1}, \mathbb{H}_{1}\right] \sim N_{3}\left(\mathbf{h}_{1}, \mathbb{H}_{1}\right),\left[\boldsymbol{\mu}_{\alpha} \mid \mathbf{h}_{2}, \mathbb{H}_{2}\right] \sim N_{2}\left(\mathbf{h}_{2}, \mathbb{H}_{2}\right),} \\
{\left[\Sigma_{\beta}^{-1} \mid v_{1}, \mathbb{A}_{1}\right] \sim W\left(v_{1},\left(v_{1} \mathbb{A}_{1}\right)^{-1}\right),\left[\Sigma_{\alpha}^{-1} \mid v_{2}, \mathbb{A}_{2}\right] \sim W\left(v_{2},\left(v_{2} \mathbb{A}_{2}\right)^{-1}\right),} \\
{\left[\sigma_{i}^{-2} \mid a_{0}, a_{1}\right] \sim G\left(\frac{a_{0}}{2}, \frac{a_{1}}{2}\right),\left[\delta \mid b_{0}, b_{1}\right] \sim U\left(b_{0}, b_{1}\right)}
\end{array}\right\},
$$

where $\left|\mathbb{I}_{m}-\delta \mathbb{W}\right|^{n}$ in (4) represents the Jacobian term taking into account the endogeneity of $\sum_{k=1}^{m} w_{i k} y_{k j}$ (Anselin, 1988, p. 63), and $g(\cdot)$ is used to denote a density function. In the hierarchical formulation of the model, we use $N_{p}$, $L N_{p}, W, G$ and $U$ to represent $p$-variate normal, $p$-variate lognormal, Wishart, gamma and uniform distributions, respectively, with all the hyperparameters assumed known. Note that $\boldsymbol{\mu}_{\beta}=\left(\mu_{0}, \mu_{1}, \mu_{2}\right)^{\prime}$ and $\Sigma_{\beta}$ are, respectively, the mean and covariance of $\boldsymbol{\beta}_{i}$, which characterize the global (population) trends in the incoming and outgoing phases: $\mu_{0}$ and $\mu_{1}$ are, respectively, the population intercept and slope for the incoming phase, $\mu_{1}+\mu_{2}$ is the population slope for the outgoing phase, and $\Sigma_{\beta}$ provides information about the variability of the linear parameters $\boldsymbol{\beta}_{i}$. Similarly, $\boldsymbol{\mu}_{\alpha}=\left(\mu_{\gamma}, \mu_{\tau}\right)^{\prime}$ characterizes the global transition. Since our assumption for $\boldsymbol{\alpha}_{i}$ involves a lognormal distribution, the medians $\mathcal{M}_{\gamma}=\exp \left\{\mu_{\gamma}\right\}$ and $\mathcal{M}_{\tau}=\exp \left\{\mu_{\tau}\right\}$ can be regarded as the half-width and the center of the bend, respectively, for the population. We can also use the standard deviations of $\gamma_{i}$ and $\tau_{i}$ to describe the between-station variability for the transition parameters.

\section{Bayesian Inference and Implementation}

Bayesian inference is based on the posterior distribution of a parameter given the data: the density function provides the behavior of the parameter, and the posterior mean (median if the posterior density is noticeably asymmetric) and variance can be considered a point estimate of the parameter and the uncertainty of the estimate, respectively. The posterior distribution of a parameter, say $\boldsymbol{\mu}_{\beta}$, denoted by $\pi\left(\boldsymbol{\mu}_{\beta} \mid \mathbf{y}\right)$, can also be used to determine points $p_{1}$ and $p_{2}$ such that $\int_{p_{1}}^{p_{2}} \pi\left(\boldsymbol{\mu}_{\beta} \mid \mathbf{y}\right) d \boldsymbol{\mu}_{\beta}=0.95$. This gives a 95\% Bayesian credible interval, $(p 1, p 2)$ for $\boldsymbol{\mu}_{\beta}$.

Bayesian inference is carried out here by Markov chain Monte Carlo (MCMC) technique. We employ the Metropolis within Gibbs algorithm (Smith \& Roberts, 1993) to approximate the posterior densities of the parameters. The algorithm is based on drawing random samples from the the full conditionals of each of the components of $\Theta$; we work out the full conditionals for each component to implement this algorithm. We have written our own code in $\mathrm{C}$ to generate MCMC samples, which we subsequently analyze using the "coda" (Plummer, Best, Cowles, \& Vines, 2006) and "MCMCpack" (Martin, Quinn, \& Park, 2011) packages in R (R Development Core Team, 2012). 


\section{CFC Data Analysis}

\subsection{The Spatial Matrix and Model Building}

The elements of the spatial matrix $\mathbb{W}$ is defined by

$$
w_{i k}= \begin{cases}1, & \text { if } i \neq k \text { and } i \sim k \\ 0, & \text { otherwise }\end{cases}
$$

where $i \sim k$ denotes the contiguity of station $i$ with station $k$. The specification of the spatial weights is an important problem and is often chosen on an ad hoc basis (Anselin, LeGallo, \& Jayet, 2008).

For the CFC data, since winds can spread CFCs across the world, there is no clearly defined boundary by which the locations of the monitoring stations can be differentiated. Therefore, we consider several models by defining the spatial configurations based on the distances between the stations, and choose the one for which the estimate of the deviance information criterion (DIC) is minimum (Spiegelhalter, Best, Carlin, \& Van der Linde, 2002). Specifically, letting $d_{i k}$ be the distance between stations $i$ and $k$, we consider the following spatial configurations:

Model 1: $\mathbb{W}=\mathbb{I}$ (i.e., no spatial effects),

Model 2: $i \sim k$ if $d_{i k} \leq 6,000 \mathrm{~km}$,

Model 3: $i \sim k$ if $d_{i k} \leq 10,000 \mathrm{~km}$,

Model 4: $i \sim k$ if $d_{i k} \leq 20,000 \mathrm{~km}$.

Note that for Model 4, $w_{i k}=1 \forall i \neq k$, hypothesizing that CFCs are distributed around the globe. Note also that we normalize $\mathbb{W}$ by dividing all of its elements by its largest characteristic root.

For MCMC, we construct two Markov chains each of 5,000, 000 iterations to approximate the posterior density. The initial 100,000 iterations are discarded as burn-in, and the inferences are based on every $200^{\text {th }}$ iteration of the chains (thinning), resulting in a total of 24,500 iterations per chain. In Table, the DICs are given for each model. The smallest DIC results from Model 4 for both CFC-11 and 12. Hence, according to the DIC values, Model 4 is estimated to be the model that would best fit the data among the models under consideration. This also supports the notion of the global distribution of the CFCs (i.e., each station is a neighbour of the remaining ones). Now, we report the results for Model 4 in Section 5.2.

\subsection{Results}

The trace and density plots for $\delta$ for both types of CFCs are presented in Figure 3. The lack of any trend in the trace plot indicates good mixing. The density plots display no signs of multimodality. These plots show stationarity of the Markov chains. We also test the stationarity of the chains for $\delta$ by the Gelman-Rubin statistic $R$ (Gelman \& Rubin, 1992) and the Geweke $Z$ score (Geweke, 1992). Values of $R$ are 1 for both CFC-11 and 12, and the $|Z|$ scores are 1.28 and 0.53 for CFC-11 and 12, respectively, none of which are greater than the critical value 1.96 at $5 \%$ level of significance. So, these two diagnostic criteria also provide no evidence against stationarity of the chains for $\delta$.
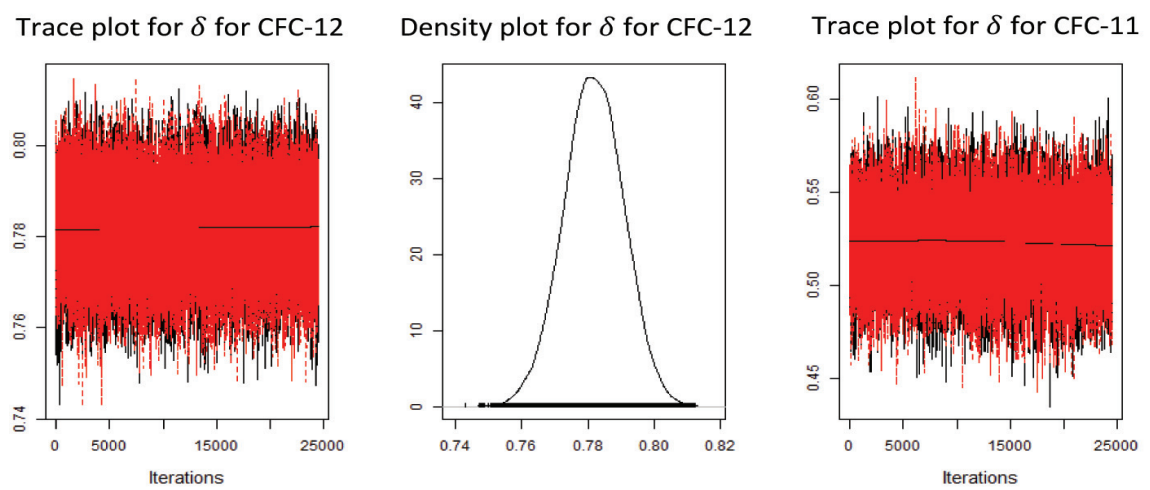

Density plot for $\delta$ for CFC-11

Figure 3. Trace and density plots for the posteriors of the spatial autocorrelation parameter $\delta$ from two chains

For CFC-12, the posterior mean for $\delta$ is 0.78 with $95 \%$ credible interval $(0.76,0.80)$, and that for CFC-11 is 0.52 
with $95 \%$ credible interval $(0.48,0.58)$. These indicate significant spatial autocorrelations for both types of CFCs, with stronger association for CFC-12. The stronger association for CFC-12 is also an evidence of its extended lifetime in the atmosphere: 45 years for CFC-11 and 100 years for CFC-12.
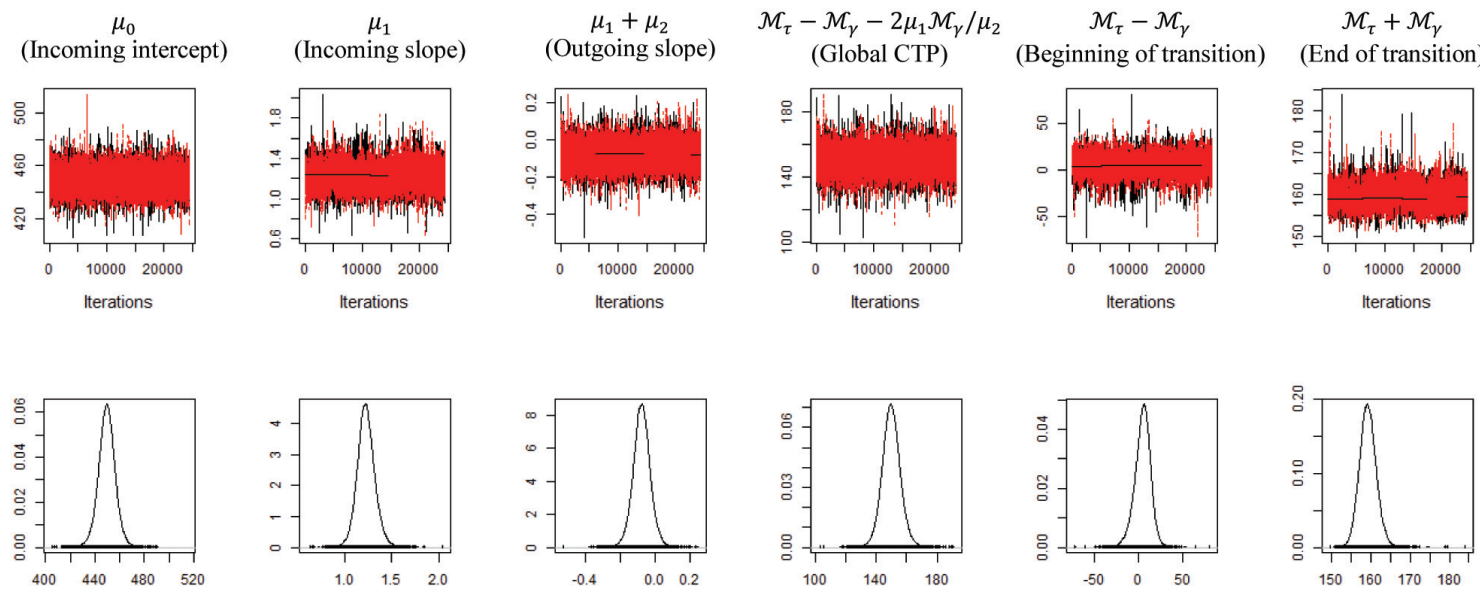

Figure 4. CFC-12 analysis-trace and density plots for the posteriors of the population parameters from two chains
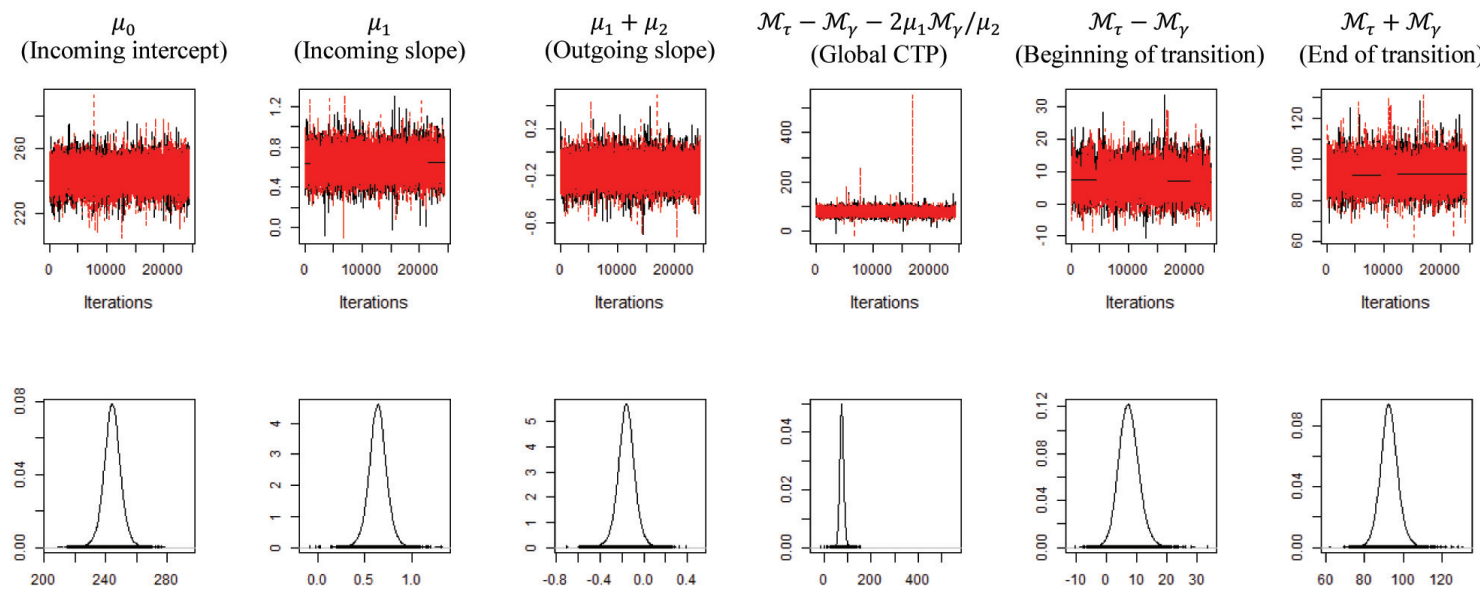

Figure 5. CFC-11 analysis-race and density plots for the posteriors of the population parameters from two chains

The trace and density plots for the population parameters of interest are displayed in Figures 4 and 5 for CFC-12 and 11, respectively. The lack of any trend in the trace plots indicate stationarity of the Markov chains. The density plots are roughly bell-shaped. Table 3 summarizes the posterior characteristics along with the convergence diagnostic criteria for the population parameters that describe the global CFC trend, and Figure 6 displays the global fits. Once again, the diagnostic criteria (Gelman-Rubin $R$ and Geweke $|Z|$ ) provide no evidence against stationarity of the chains. From Table 3, we see that both CFC-11 and CFC-12 increased significantly (95\% credible intervals for $\mu_{1}$ are $(0.45,0.83)$ and $(1.04,1.41)$, respectively, none of which includes zero) prior to the transition phase, with the increase for CFC-12 occurred at a faster rate $\left(\hat{\mu}_{1}=1.23\right)$ than that for CFC-11 $\left(\hat{\mu}_{1}=0.64\right)$. After the initial increase, a transition took place. Although the transition for both types of CFCs began at approximately the same time (08/1988 for CFC-11 and 05/1988 for CFC-12), it took longer for CFC-12 to complete the transition: around 155 months for CFC-12 (05/1988 to 04/2001), and 85 months for CFC-11 (08/1988 to 09/1995). Overall, the concentration of CFC-11 took a downturn from an increasing trend at around $\overline{\mathrm{CTP}}=05 / 1994$ (95\% credible interval ranges from $11 / 1992$ to $10 / 1995)$, whereas this downturn for CFC-12 took place at around $\widehat{\mathrm{CTP}}=05 / 2000$ (95\% credible interval ranges from 06/1999 to 06/2001). After completing the transition, CFC-11 decreased significantly $\left(\widehat{\mu_{1}+\mu_{2}}=-0.16\right.$ with $95 \%$ credible interval $\left.(-0.313,-0.002)\right)$, whereas an insignificant decrease for CFC-12 was observed $\widehat{\left(\widehat{\mu_{1}+\mu_{2}}\right.}=-0.08$ with $95 \%$ credible interval $\left.(-0.18,0.02)\right)$. Notwithstanding potentially 
differential adverse health effects for a given amount of CFC-12 vs. CFC-11, the above results indicate a much more severe global concern for CFC-12 comapred to CFC-11 with respect to their presence in the atmosphere. The posterior means of the intercept parameter $\left(\mu_{0}\right)$ also suggests that the atmospheric concentration of CFC-12 is much higher than that of CFC-11 ( $\hat{\mu}_{0}=449.62$ and 244.28 for CFC-12 and CFC-11, respectively). This is also evident from the scales of the y-axes in Figure 6-CFC-12 ranges from 414-548 ppt, whereas it is $227-280$ ppt for CFC-11. In fact, the rates by which CFCs have been decreasing suggest that it may take almost 5 times longer to diminish CFC-12 from the atmosphere compared to CFC-11, should current conditions prevail.

Table 3. Posterior summaries of the global regression parameters

\begin{tabular}{|c|c|c|c|c|c|c|}
\hline & \multicolumn{3}{|c|}{ CFC-12 } & \multicolumn{3}{|c|}{ CFC-11 } \\
\hline & \multirow{2}{*}{$\begin{array}{c}\text { Posterior mean } \\
\text { (95\% credible interval) }\end{array}$} & \multicolumn{2}{|c|}{ Convergence diagnostics } & \multirow{2}{*}{$\begin{array}{c}\text { Posterior mean } \\
\text { (95\% credible interval) }\end{array}$} & \multicolumn{2}{|c|}{ Convergence diagnostics } \\
\hline & & Gelman-Rubin $R$ & Geweke $|Z|$ & & Gelman-Rubin $R$ & Geweke $|Z|$ \\
\hline $\begin{array}{c}\mu_{0} \\
\text { (Incoming intercept) }\end{array}$ & $\begin{array}{c}449.62 \\
(435.98,463.47)\end{array}$ & 1.00 & 0.04 & $\begin{array}{c}244.28 \\
(233.28,255.62)\end{array}$ & 1.00 & 0.76 \\
\hline $\begin{array}{c}\mu_{1} \\
\text { (Incoming slope) }\end{array}$ & $\begin{array}{c}1.23 \\
(1.04,1.41)\end{array}$ & 1.00 & 0.98 & $\begin{array}{c}0.64 \\
(0.45,0.83)\end{array}$ & 1.00 & 0.62 \\
\hline $\begin{array}{c}\mu_{1}+\mu_{2} \\
\text { (Outgoing slope) }\end{array}$ & $\begin{array}{c}-0.08 \\
(-0.18,0.02)\end{array}$ & 1.00 & 0.44 & $\begin{array}{c}-0.16 \\
(-0.313,-0.002)\end{array}$ & 1.00 & 0.64 \\
\hline $\begin{array}{c}\mathcal{M}_{\tau}-\mathcal{M}_{\gamma}-2 \mu_{1} \mathcal{M}_{\gamma} / \mu_{2} \\
\text { (Global CTP) }\end{array}$ & $\begin{array}{c}06 / 2000 \\
(06 / 1999 \text { to } 06 / 2001)\end{array}$ & 1.00 & 1.41 & $\begin{array}{c}05 / 1994 \\
(11 / 1992 \text { to } 10 / 1995)\end{array}$ & 1.00 & 0.48 \\
\hline $\begin{array}{l}\mathcal{M}_{\tau}-\mathcal{M}_{\gamma} \text { to } \mathcal{M}_{\tau}+\mathcal{M}_{\gamma} \\
\quad \text { (Transition period) }\end{array}$ & $\begin{array}{c}05 / 1988 \text { to } 04 / 2001 \\
-\end{array}$ & $\begin{array}{c}1.00,1.01 \\
-\end{array}$ & $\begin{array}{c}0.99,0.93 \\
-\end{array}$ & $\begin{array}{c}08 / 1988 \text { to } 09 / 1995 \\
-\end{array}$ & $\begin{array}{c}1.00,1.00 \\
-\end{array}$ & $\begin{array}{c}0.91,0.68 \\
-\end{array}$ \\
\hline
\end{tabular}

(a) CFC-12

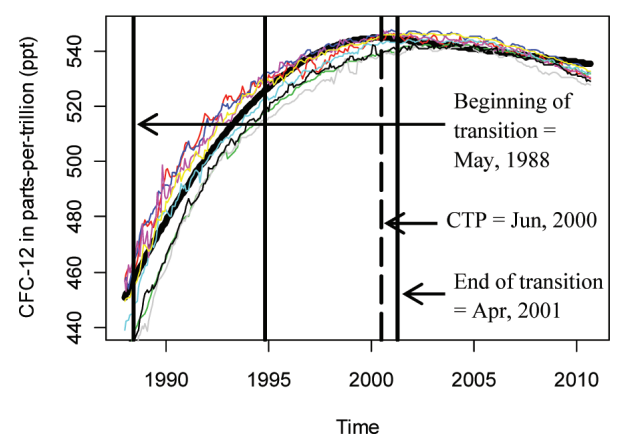

(b) CFC-11

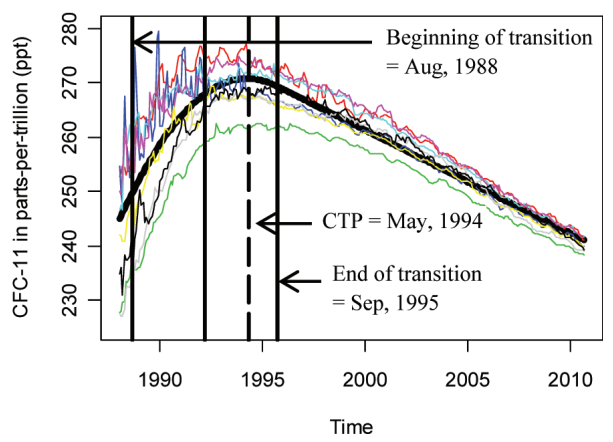

Figure 6. Observed trajectories for the CFC data with fitted global curves (solid, in black). The estimated transition, $\widehat{\mathcal{M}_{\tau} \pm \mathcal{M}_{\gamma}}$, is marked by the solid black vertical lines, and the estimated CTP is indicated by the dotted vertical line

We now turn to the behaviour of the station-specific trajectories described by the regression coefficients $\boldsymbol{\beta}_{i}$ and $\boldsymbol{\alpha}_{i}$, and the between-station variability characterized by $\Sigma_{\beta}$ and $\Sigma_{\alpha}$. The trace plots and convergence diagnostic criteria (not shown) provide no evidence against the stationarity of the chains for $\boldsymbol{\beta}_{i}, \boldsymbol{\alpha}_{i}, \Sigma_{\beta}$ and $\Sigma_{\alpha}$. Table 4 summarizes the posterior characteristics of the station-specific fits, and Figure 7 displays one representative fitted curve for each of CFC-11 and CFC-12. The figure shows that our model fits the data well, with the observed data and individual fits agreeing quite closely. All the station sites exhibit significant increase of both types of CFCs before the transition took place. The rate of increase was maximum in South Pole $\left(\hat{\beta}_{1}=1.49\right.$ and 0.98 for CFC-12 and CFC-11, respectively). The transition ended in around 2001 for CFC-12, and between 07/1994 and 12/1996 for CFC-11. After completing the transition, CFC-11 decreased at a higher rate across all stations. The most rapid decrease took place in Niwot Ridge $\left(\widehat{\beta_{1}+\beta_{2}}=-0.11\right.$ and -0.178 for CFC-12 and CFC-11, respectively). Posterior characteristics of the standard deviations describing the between-station variability are summarized in Table 5 . We see small variation in the slope parameters $\beta_{1 i}$ and $\beta_{2 i}$ across the stations. This suggests that the rates at which CFCs increased and decreased in the incoming and outgoing phases are similar across stations. However, we see some variation for the transition parameters $\gamma_{i}$ and $\tau_{i}$ (i.e., in the times to transition zones). 
Table 4. Posterior summaries of the station-specific regression parameters

\begin{tabular}{|c|c|c|c|c|c|c|}
\hline Station & $\begin{array}{c}\beta_{1 i} \\
\text { (95\% credible } \\
\text { interval) } \\
\end{array}$ & $\begin{array}{c}\text { CFC-12 } \\
\beta_{1 i}+\beta_{2 i} \\
(95 \% \text { credible } \\
\text { interval })\end{array}$ & $\tau_{i} \pm \gamma_{i}$ & $\begin{array}{c}\beta_{1 i} \\
\text { (95\% credible } \\
\text { interval) } \\
\end{array}$ & $\begin{array}{c}\text { CFC-11 } \\
\beta_{1 i}+\beta_{2 i} \\
(95 \% \text { credible } \\
\text { interval })\end{array}$ & $\tau_{i} \pm \gamma_{i}$ \\
\hline Barrow & $\begin{array}{c}1.09 \\
(0.98,1.23)\end{array}$ & $\begin{array}{c}-0.09 \\
(-0.10,-0.08)\end{array}$ & $11 / 1987$ to $03 / 2001$ & $\begin{array}{c}0.64 \\
(0.58,0.71)\end{array}$ & $\begin{array}{c}-0.168 \\
(-0.171,-0.165)\end{array}$ & $06 / 1988$ to $07 / 1994$ \\
\hline Cape Grim & $\begin{array}{c}1.42 \\
(1.30,1.55)\end{array}$ & $\begin{array}{c}-0.05 \\
(-0.06,-0.04)\end{array}$ & $11 / 1987$ to $04 / 2001$ & $\begin{array}{c}0.74 \\
(0.67,0.82)\end{array}$ & $\begin{array}{c}-0.137 \\
(-0.139,-0.134)\end{array}$ & $11 / 1988$ to $02 / 1996$ \\
\hline Mace Head & $\begin{array}{c}1.16 \\
(1.06,1.29)\end{array}$ & $\begin{array}{c}-0.09 \\
(-0.10,-0.08)\end{array}$ & $08 / 1987$ to $04 / 2001$ & $\begin{array}{c}0.43 \\
(0.34,0.52)\end{array}$ & $\begin{array}{c}-0.148 \\
(-0.154,-0.142)\end{array}$ & $07 / 1988$ to $10 / 1994$ \\
\hline Mauna Loa & $\begin{array}{c}1.35 \\
(1.17,1.54)\end{array}$ & $\begin{array}{c}-0.08 \\
(-0.09,-0.07)\end{array}$ & $05 / 1986$ to $12 / 2001$ & $\begin{array}{c}0.41 \\
(0.37,0.45)\end{array}$ & $\begin{array}{c}-0.172 \\
(-0.176,-0.168)\end{array}$ & $04 / 1988$ to $12 / 1996$ \\
\hline Niwot Ridge & $\begin{array}{c}0.87 \\
(0.83,0.91)\end{array}$ & $\begin{array}{c}-0.11 \\
(-0.12,-0.10)\end{array}$ & $07 / 1991$ to $04 / 2001$ & $\begin{array}{c}0.48 \\
(0.42,0.53)\end{array}$ & $\begin{array}{c}-0.178 \\
(-0.182,-0.174)\end{array}$ & $08 / 1988$ to $09 / 1996$ \\
\hline Ragged Point & $\begin{array}{c}1.23 \\
(1.11,1.37)\end{array}$ & $\begin{array}{c}-0.09 \\
(-0.09,-0.08)\end{array}$ & $01 / 1988$ to $04 / 2001$ & $\begin{array}{c}0.67 \\
(0.61,0.74)\end{array}$ & $\begin{array}{c}-0.146 \\
(-0.149,-0.143)\end{array}$ & $06 / 1988$ to $04 / 1995$ \\
\hline South Pole & $\begin{array}{c}1.49 \\
(1.35,1.66)\end{array}$ & $\begin{array}{c}-0.07 \\
(-0.08,-0.06)\end{array}$ & $09 / 1987$ to $06 / 2001$ & $\begin{array}{c}0.98 \\
(0.90,1.07)\end{array}$ & $\begin{array}{c}-0.155 \\
(-0.159,-0.151)\end{array}$ & $08 / 1988$ to $06 / 1995$ \\
\hline Cape Matatula & $\begin{array}{c}1.21 \\
(1.16,1.26)\end{array}$ & $\begin{array}{c}-0.06 \\
(-0.07,-0.05)\end{array}$ & $12 / 1989$ to $01 / 2001$ & $\begin{array}{c}0.73 \\
(0.64,0.84)\end{array}$ & $\begin{array}{c}-0.161 \\
(-0.165,-0.158)\end{array}$ & $02 / 1989$ to $03 / 1996$ \\
\hline
\end{tabular}

(a) CFC-12 for Barrow, Alaska

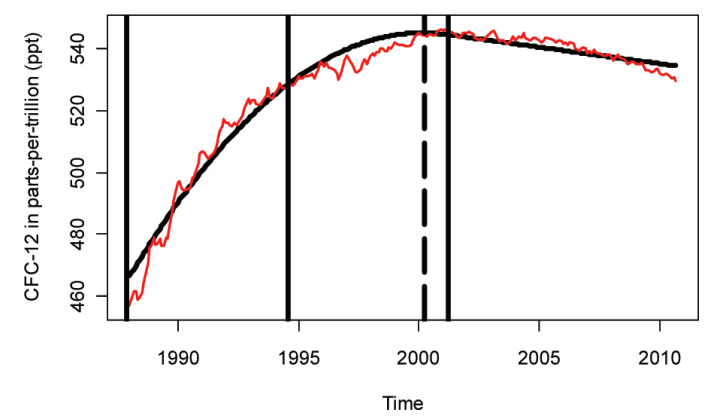

(b) CFC-11 for Barrow, Alaska

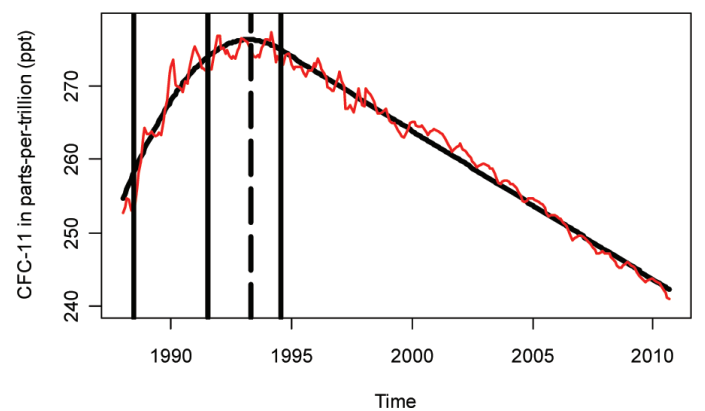

Figure 7. Observed trajectories (red curves) and the corresponding station-specific fitted curves (solid, in black) for a representative monitoring station, Barrow, Alaska. Estimated transitions (i.e., $\widehat{\tau \pm \gamma}$ and $\hat{\tau}$ ) are marked by solid vertical lines, and the estimated CTPs by dotted vertical lines

Table 5. Posterior summaries of the standard deviations of the random regression coefficients

\begin{tabular}{|c|c|c|}
\hline & $\begin{array}{c}\text { CFC-12 } \\
\text { Posterior mean } \\
(95 \% \text { credible interval })\end{array}$ & $\begin{array}{c}\text { CFC-11 } \\
\text { Posterior mean } \\
(95 \% \text { credible interval })\end{array}$ \\
\hline $\operatorname{SD}\left(\beta_{0 i}\right)$ & $\begin{array}{c}18.77 \\
(10.50,29.75)\end{array}$ & $\begin{array}{c}15.18 \\
(8.29,23.89)\end{array}$ \\
\hline $\operatorname{SD}\left(\beta_{1 i}\right)$ & $\begin{array}{c}0.24 \\
(0.12,0.38)\end{array}$ & $\begin{array}{c}0.25 \\
(0.14,0.40)\end{array}$ \\
\hline $\operatorname{SD}\left(\beta_{2 i}\right)$ & $\begin{array}{c}0.22 \\
(0.11,0.36)\end{array}$ & $\begin{array}{c}0.25 \\
(0.13,0.39)\end{array}$ \\
\hline $\mathrm{SD}\left(\gamma_{i}\right)$ & $\begin{array}{c}11.63 \\
(5.40,21.58)\end{array}$ & $\begin{array}{c}6.45 \\
(3.42,11.59)\end{array}$ \\
\hline $\mathrm{SD}\left(\tau_{i}\right)$ & $\begin{array}{c}8.87 \\
(4.36,16.51)\end{array}$ & $\begin{array}{c}7.19 \\
(3.83,12.83)\end{array}$ \\
\hline
\end{tabular}

In summary, our analysis reveals the following points of interest: (1) strong spatial dependence among all stations suggest that both CFC-11 and CFC-12 have already been distributed globally; (2) both types of CFCs increased significantly before entering into a transition zone, though CFC-12 increased at a faster rate compared to the 
increase of CFC-11; (3) on average, CFC-11 completed the transition between 08/1988 and 09/1995, whereas CFC12 between 05/1988 and 04/2001; (4) CFC-11 has been decreasing significantly after completing the transition, but the rate at which CFC-12 has been decreasing is not significant; and (5) although the rates of increase and decrease are similar across stations for each type of $\mathrm{CFC}$, there are considerable variations in the times to transition zone. Though the two types of CFCs may pose potentially differential health effects, the above findings also indicate a much more severe global concern for CFC-12 with respect to its concentration in the atmosphere.

\section{Simulation}

In this section, we present a simulation study to demonstrate the efficacy of our spatial-longitudinal bent-cable methodology. In particular, we supplement the motivation of our methodology by illustrating the importance of properly taking into account the spatial component. To this end, we present a scenario, where, in reality, spatial dependency exists among the units. We then analyze the data using the true model, as well as models with misspecified spatial configuration.

Model parameter values in the simulations are chosen to approximately mimic the CFC-11 data. We take $m=8$, $n=273$ and $t_{i j}=j-1$ for $i=1,2, \ldots, m$ and $j=1,2, \ldots, n$. We generate data assuming Model 4 (see Section 5.1) with $\delta=0.52$. We also take $\boldsymbol{\mu}_{\beta}=(244.28,0.64,-0.79)^{\prime}, \boldsymbol{\mu}_{\alpha}=(3.75,3.91)^{\prime}$,

$$
\Sigma_{\beta}=\left[\begin{array}{rrr}
204.66 & -2.46 & 2.35 \\
-2.46 & 0.06 & -0.04 \\
2.35 & -0.04 & 0.05
\end{array}\right], \quad \Sigma_{\alpha}=\left[\begin{array}{ll}
0.022 & 0.012 \\
0.012 & 0.020
\end{array}\right],
$$

and $\sigma_{i}^{2}$ 's are 1.36, 0.63, 6.08, 1.63, 1.54, 1.16, 1.89, and 1.39. Given the parameters, we generate $\boldsymbol{\beta}_{i}$ 's and $\boldsymbol{\alpha}_{i}$ 's from $N_{3}\left(\boldsymbol{\mu}_{\beta}, \Sigma_{\beta}\right)$ and $L N_{2}\left(\boldsymbol{\mu}_{\alpha}, \Sigma_{\alpha}\right)$, respectively. Then, $y_{i j}$ 's are generated from (4) using Metropolis-Hastings algorithm. We analyze the data using Models 1 (no spatial dependency), 2 (misspecified spatial configuration), and 4 (true model) to evaluate the performance of our methodology and the effects of misspecified spatial configuration.

For each simulation, 500 data sets are generated, and 10,000 MCMC iterations are used to approximate posterior distributions per set. DICs and posterior summaries for each parameter are then averaged over the 500 sets. In addition to the DICs, relative mean square error (RMSE) of the posterior summaries is used to evaluate the performance of the analytic procedures. RMSE takes into account both bias (measured by the relative bias, RB) and variability (gauged by the relative standard deviation, RSD), according to the formula $R M S E=R B^{2}+R_{S D}^{2}$, where $\mathrm{RB}=$ bias/true parameter value, $\mathrm{RSD}=\mathrm{SD} /$ true parameter value, and $\mathrm{SD}$ is the standard deviation of the posterior means/medians across the 500 simulated data sets. Note that the smaller the RMSE, the more accurate an estimator is.

Table 6. Simulation results for data generated from Model 4 with $n=273$ and $m=8$ : absolute relative bias (RB), absolute relative standard deviation (RSD) and relative mean square error (RMSE) of the population regression coefficients and the spatial autocorrelation coefficient, calculated by considering average of 500 posterior means

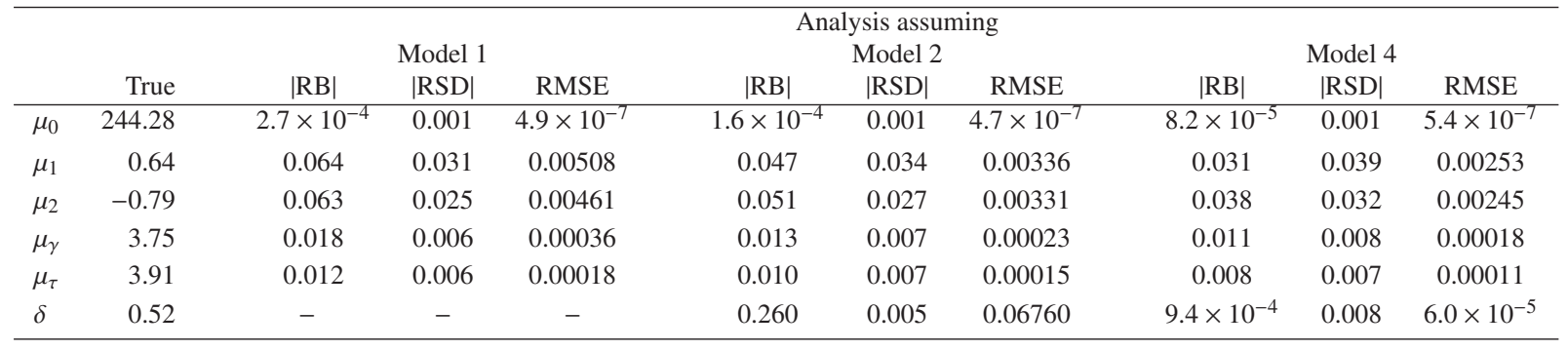

Numerical results for the population regression coefficients and spatial autocorrelation parameter are summarized in Table 6. For $\mu_{0}$, both $|R B|$ and RMSE are very close to zero regardless of the assumption about the spatial configuration. Smallest $|R B|$ and RMSE for each of the other parameters are observed when we analyze using the true model (i.e., Model 4), whereas the largest $|R B|$ and RMSE are observed if we completely ignore spatial dependency (i.e., Model 1). Similar results are obtained for $\sigma_{i}^{2}$ 's (Table 7), and $\Sigma_{\beta}$ and $\Sigma_{\alpha}$ (Table 7), that is, the smallest $|R B|$ and RMSE occur when we analyze using the true model, and the largest $|R B|$ and RMSE if we ignore spatial dependency. The only exception occurs for $\left(\Sigma_{\alpha}\right)_{12}$ (Table 7), for which the smallest $|R B|$ and RMSE are observed for Model 2. The average DICs are 7893, 7720, and 7379 for Models 1, 2 and 4, respectively. This 
indicates that the best fit is achieved by Model 4 (smallest DIC), and a comparatively poor fit if we completely ignore the spatial dependency (largest DIC).

Table 7. Simulation results for data generated from Model 4 with $n=273$ and $m=8$ : absolute relative bias (RB), absolute relative standard deviation (RSD) and relative mean square error (RMSE) of $\sigma_{i}^{2}$ 's, calculated by considering average of 500 posterior medians

\begin{tabular}{ccccccccccc}
\hline & \multicolumn{3}{c}{ Model 1 } & \multicolumn{9}{c}{ Analysis assuming } \\
& True & $\mid$ RB $\mid$ & $\mid$ RSD $\mid$ & RMSE & $\mid$ RB $\mid$ & $\mid$ RSD $\mid$ & RMSE & $\mid$ RB $\mid$ & Model 4 \\
& $\mid$ RSD $\mid$ & RMSE \\
\hline$\sigma_{1}^{2}$ & 1.36 & 0.3281 & 0.0071 & 0.10770 & 0.1058 & 0.0060 & 0.01123 & 0.0063 & 0.0064 & 0.00008 \\
$\sigma_{2}^{2}$ & 0.63 & 0.6909 & 0.0091 & 0.47747 & 0.0427 & 0.0081 & 0.00189 & 0.0063 & 0.0073 & 0.00009 \\
$\sigma_{3}^{2}$ & 6.08 & 0.1234 & 0.0062 & 0.01528 & 0.0643 & 0.0064 & 0.00418 & 0.0031 & 0.0058 & 0.00004 \\
$\sigma_{4}^{2}$ & 1.63 & 0.3711 & 0.0074 & 0.13777 & 0.1239 & 0.0092 & 0.01543 & 0.0092 & 0.0097 & 0.00018 \\
$\sigma_{5}^{2}$ & 1.54 & 0.2809 & 0.0064 & 0.07896 & 0.0188 & 0.0059 & 0.00039 & 0.0003 & 0.0057 & 0.00003 \\
$\sigma_{6}^{2}$ & 1.16 & 0.3465 & 0.0071 & 0.12014 & 0.2317 & 0.0067 & 0.05374 & 0.0013 & 0.0062 & 0.00004 \\
$\sigma_{7}^{2}$ & 1.89 & 0.5129 & 0.0078 & 0.26313 & 0.2838 & 0.0074 & 0.08057 & 0.0054 & 0.0069 & 0.00008 \\
$\sigma_{8}^{2}$ & 1.39 & 0.2114 & 0.0068 & 0.04473 & 0.0996 & 0.0056 & 0.00995 & 0.0080 & 0.0068 & 0.00011 \\
\hline
\end{tabular}

Table 8. Simulation results for data generated from Model 4 with $n=273$ and $m=8$ : absolute relative bias (RB), absolute relative standard deviation (RSD) and relative mean square error (RMSE) of the covariance matrices $\Sigma_{\beta}$ and $\Sigma_{\alpha}$, calculated by considering average of 500 posterior means for the covariances $\left(\Sigma_{\beta}\right)_{i j}$ and $\left(\Sigma_{\alpha}\right)_{i j}$ for $i \neq j$, and average of 500 posterior medians for the variances $\left(\Sigma_{\beta}\right)_{i i}$ and $\left(\Sigma_{\alpha}\right)_{i i}$

\begin{tabular}{rrrrrcccccc}
\hline & \multicolumn{1}{c}{ Model 1 } & \multicolumn{4}{c}{ Analysis assuming } & \multicolumn{3}{c}{ Model 2} \\
& True & $\mid$ RB $\mid$ & $\mid$ RSD $\mid$ & RMSE & $\mid$ RB $\mid$ & $\mid$ RSD $\mid$ & RMSE & $\mid$ RB $\mid$ & $\mid$ RSD $\mid$ & RMSE \\
\hline$\left(\Sigma_{\beta}\right)_{11}$ & 204.660 & 0.116 & 0.019 & 0.014 & 0.116 & 0.020 & 0.014 & 0.114 & 0.020 & 0.013 \\
$\left(\Sigma_{\beta}\right)_{22}$ & 0.060 & 0.373 & 0.114 & 0.152 & 0.352 & 0.135 & 0.142 & 0.311 & 0.143 & 0.117 \\
$\left(\Sigma_{\beta}\right)_{33}$ & 0.050 & 0.487 & 0.132 & 0.254 & 0.467 & 0.159 & 0.243 & 0.428 & 0.167 & 0.211 \\
$\left(\Sigma_{\beta}\right)_{12}$ & -2.460 & 0.099 & 0.086 & 0.017 & 0.095 & 0.099 & 0.019 & 0.050 & 0.115 & 0.016 \\
$\left(\Sigma_{\beta}\right)_{13}$ & 2.350 & 0.099 & 0.089 & 0.018 & 0.095 & 0.103 & 0.020 & 0.049 & 0.121 & 0.017 \\
$\left(\Sigma_{\beta}\right)_{23}$ & -0.040 & 0.267 & 0.167 & 0.099 & 0.240 & 0.200 & 0.097 & 0.185 & 0.210 & 0.078 \\
$\left(\Sigma_{\alpha}\right)_{11}$ & 0.022 & 0.621 & 0.359 & 0.515 & 0.378 & 0.342 & 0.260 & 0.255 & 0.306 & 0.159 \\
$\left(\Sigma_{\alpha}\right)_{22}$ & 0.020 & 0.382 & 0.356 & 0.273 & 0.250 & 0.352 & 0.187 & 0.165 & 0.312 & 0.125 \\
$\left(\Sigma_{\alpha}\right)_{12}$ & 0.012 & 0.647 & 0.277 & 0.495 & 0.165 & 0.289 & 0.111 & 0.252 & 0.312 & 0.161 \\
\hline
\end{tabular}

The above simulation results demonstrate the importance of modelling the spatial component when, in reality, spatial dependency exists among the units. Moreover, smaller bias and highly accurate (smaller RMSE) estimates indicate that our proposed methodology performs well to analyze data that resemble those from the CFC study.

\section{Conclusion}

Under a spatially dependent process, each unit is typically affected by those of the neighbouring units. Therefore, the assumption of independence across the neighbouring units might be unrealistic, especially when they represent geographical locations. Moreover, addressing the spatial effects provide important insights about such a process. In this article, we propose an extension of the longitudinal bent-cable model by taking into account spatial effects. We have tailored our work especially for the scientific context of the CFC data. Due to their extended lifetimes, CFCs persist long enough in the atmosphere, and consequently are believed to have spread across the world. Therefore, CFCs monitored from one station may depend on those from another station, giving rise to a presumed spatially dependent longitudinal process. Our methodology provides understanding not only of the spatial distribution, but also of the global threat that CFCs may pose to all living organisms. It also reveals useful information regarding the atmospheric CFC decline throughout the globe.

Since the Montréal protocol came into effect, a global decrease in the CFCs is monitored and confirmed by our analysis. Note that the Montréal Protocol contains an extended CFC phase-out schedules-1996 for developed countries and 2010 for developing countries. Thus, many countries at various geographical locations continued to contribute CFCs to the atmosphere during the 273 months in our study period. In our analysis, this fact is reflected with a slow decrease in CFC concentrations from the atmosphere. In fact, our analysis does not reveal a 
significant decrease for CFC-12. This makes sense due to the extended lifetime for CFC-12 comapred to CFC-11. Nevertheless, the Montréal protocol can be regarded as a successful international agreement to reduce the use of the CFCs, in particular, thus far, for CFC-11.

There are other modeling approaches that can be used to characterize non-linear trend change over time. For example, a piecewise linear model with three knots could be fit, where the transition period for the bent cable model would instead be approximated by two straight lines with one internal knot. In case a piecewise linear model with three knots would not fit the data well, another model could be fit with, say, five knots (three inside the transition, instead of one). This process may lead to investigating many models, and, due to the smooth transition phase, at least for the CFC data, may lead to too many parameters, which might diminish interpretability as compared to the bent-cable model. Another modeling approach for such data is the functional mixed effects model (e.g., Baladandayuthapani et al., 2008). So, further investigation is necessary to compare our methodology with other available statistical techniques to model this type of longitudinal changepoint data. Nevertheless, our proposed methodology is appealing for its many attractive features (great interpretability, flexibility and parsimony), and, as demonstrated in Section 5, it fits the CFC data well.

Although we have tailored our work for the CFC data, our modeling framework may be applicable to a wide variety of other situations across the range of the econometrics, transportation, social, health and medical sciences. However, it may be desirable to extend our framework in some cases. For example, it might be useful to consider interaction between temporal and spatial effects. With the current model, this could be done with a spatial correlation structure that changes as a function of time.

\section{Acknowledgements}

The authors extend their appreciation to the NOAA/GMDD and GAGE/AGAGE global network program, who made their CFC data available to the public. The authors would like to thank the referees for their valuable comments and suggestions.

\section{References}

Anselin, L. (1988). Spatial Econometrics: Methods and Models. Kluwer Academic, Dordrecht.

Anselin, L., Le Gallo, J., \& Jayet, H. (2008). Spatial Panel Econometrics. In M. Laszlo, \& S. Patrick (Eds.), The Econometrics of Panel Data: Fundamentals and Recent Developments in Theory and Practice (pp. 625-660). Berlin Heidelberg New York: Springer.

Baladandayuthapani, V., Mallick, B. K., Hong, M. Y., Lupton, J. R., Turner, N. D., \& Carroll, R. J. (2008). Bayesian Hierarchical Spatially Correlated Functional Data Analysis with Application to Colon Carcinogenesis. Biometrics, 64(1), 64-73. http://dx.crossref.org/10.1111\%2Fj.1541-0420.2007.00846.x

Chiu, G. S., \& Lockhart, R. A. (2010). Bent-Cable Regression with Autoregressive Noise. The Canadian Journal of Statistics, 38(3), 386-407. http://dx.doi.org/10.1002/cjs.10070

Chiu, G., Lockhart, R., \& Routledge, R. (2006). Bent-Cable Regression Theory and Applications. Journal of the American Statistical Association, 101, 542-553. http://dx.doi.org/10.1198/016214505000001177

Elhorst, J. P. (2010). Spatial Panel Data Models. In M. M. Fischer, \& A. Getis (Eds.), Handbook of Applied Spatial Analysis (pp. 377-407). Berlin Heidelberg New York: Springer. http://dx.doi.org/10.1007/978-3-642-03647-7_19

Gelman, A., \& Rubin, D. B. (1992). Inference from Iterative Simulation using Multiple Sequences. Statistical Science, 7, 457-472. http://dx.doi.org/10.1214/ss/1177011136

Geweke, J. (1992). Evaluating the Accuracy of Sampling-based Approaches to Calculating Posterior Moments. In J. M. Bernado, J. O. Berger, A. P. Dawid, \& A. F. M. Smith (Eds.), Bayesian Statistics, 4 (pp. 169-193). Oxford, UK: Clarendon Press.

Han, W., Kennedy, E. M., Mackie, J. C., \& Dlugogorski, B. Z. (2010). Conversion of a CFCs, HFCs and HCFCs Waste Mixture via Reaction with Methane. Journal of Hazardous Materials, 184(1-3), 696-703. http://dx.doi.org/10.1016/j.jhazmat.2010.08.095

Khan, S. A., Chiu, G., \& Dubin, J. A. (2009). Atmospheric Concentration of Chloroflurocarbons: Addressing the Global Concern with the Longitudinal Bent-Cable Model. CHANCE, 22(3), 8-17. http://dx.doi.org/10.1007/s00144-009-0026-x 
Martin, A. D., Quinn, K. V., \& Park, J. H. (2011). MCMCpack: Markov Chain Monte Carlo in R. Journal of Statistical Software, 42(9), 1-21. http://www.jstatsoft.org/v42/i09/

Molina, M. J., \& Rowland, F. S. (1974). Stratospheric Sink for Chlorofluoromethanes: Chlorine Atom-Catalysed Destruction of Ozone. Nature, 249, 810-812. http://dx.doi.org/10.1038/249810a0

Moulijn, J. A., Makkee, M., Wiersma, A., \& van de Sandt, E. J. A. X. (2000). Selective Hydrogenolysis of $C C l_{2} F_{2}$ into $\mathrm{CH}_{2} \mathrm{~F}_{2}$ Over Palladium on Activated Carbon: Kinetic Mechanism and Process Design. Catalysis Today, 59(3-4), 221-230. http://dx.doi.org/10.1016/S0920-5861(00)00288-1

Plummer, M., Best, N., Cowles, K., \& Vines, K. (2006). CODA: Convergence Diagnosis and Output Analysis for MCMC. R News, 6(1), 7-11. http://CRAN.R-project.org/doc/Rnews/Rnews_2006-1.pdf

Prinn, R. G., Weiss, R. F., Fraser, P. J., Simmonds P. G., Cunnold, D. M., Alyea, F. N., ... McCulloch, A. (2000). A History of Chemically and Radiatively Important Gases in Air deduced from ALE/GAGE/AGAGE. Journal of Geophysical Research, 17751-17792. http://dx.doi.org/10.1029/2000JD900141

R Development Core Team (2012). R: A Language and Environment for Statistical Computing. R Foundation for Statistical Computing, Vienna, Austria, ISBN 3-900051-07-0. http://www.R-project.org

Ravishankara, A. R., Kurylo, M. J., Bevilacqua, R., Cohen, J., Daniel, J. S., Douglass, A. R., ... Vick, K. (2008). Executive Summary. In A. R. Ravishankara, M. J. Kurylo, \& C. A. Ennis (Eds.), Trends in Emissions of Ozone-Depleting Substances, Ozone Layer Recovery, and Implications for Ultraviolet Radiation Exposure (pp. 15-22). A Report by the U.S. Climate Change Science Program and the Subcommittee on Global Change Research, Department of Commerce, NOAA's National Climatic Data Center, Asheville, NC.

Smith, A. F. M., \& Roberts, G. O. (1993). Bayesian Computation Via Gibbs Sampler and Related Markov Chain Monte Carlo Methods. Journal of the Royal Statistical Society, Series B, 55, 3-23. http://www.jstor.org/stable/2346063

Spiegelhalter, D. J., Best, N. G., Carlin, B. P., \& Van der Linde, A. (2002). Bayesian Measures of Model Complexity and Fit (with Discussion). Journal of the Royal Statistical Society, Series B, 64(4), 583-616 http://dx.doi.org/10.1111/1467-9868.00353

Struijs, J., van Dijk, A., Slaper, H., van Wijnen, H. J., Velders, G. J. M., Chaplin, G., \& Huijbregts, M. A. J (2010). Spatial- and Time-Explicit Human Damage Modeling of Ozone Depleting Substances in Life Cycle Impact Assessment. Environmental Science \& Technology, 44(1), 204-209. http://dx.doi.org/10.1021/es9017865

World Meteorological Organisation (2011). Scientice Assessment of Ozone Depletion: 2010, Global Ozone Research and Monitoring Project-Report, No. 52, Geneva, Switzerland.

Zhang, F., Zhou, L., Yao, B., Vollmer, M. K., Greally, B. R., Simmonds, P. G., ... Zhang, X. (2010). Analysis of 3-year Observations of CFC-11, CFC-12 and CFC-113 from a Semi-Rural Site in China. Atmospheric Environment, 44(35), 4454-4462. http://dx.doi.org/10.1016/j.atmosenv.2010.07.041 